\title{
Bi-univalent properties for certain class of Bazilevič functions defined by convolution and with bounded boundary rotation
}

Mohamed K. Aouf ${ }^{*}$, Samar M. Madian² and Adela O. Mostafa ${ }^{1}$

\section{*Correspondence:}

mkaouf127@yahoo.com

${ }^{1}$ Faculty of Science, Department of Mathematics, Mansoura University,

Mansoura 35516, Egypt

Full list of author information is

available at the end of the article

\begin{abstract}
In this paper, we obtain bi-univalent properties for certain class of Bazilevič functions defined by convolution and with bounded boundary rotation. We will find coefficient bounds for $\left|a_{2}\right|$ and $\left|a_{3}\right|$ for the class $\mathcal{M}_{\alpha, \lambda, \rho, k, \beta}(f * h)$.
\end{abstract}

Keywords: Bi-univalent, Bazilevič functions, Hadamard product, Bounded boundary rotation

2010 Mathematics Subject Classification: 30C45, 30C50

\section{Introduction}

Let $\mathcal{A}$ denote the class of analytic functions of the form:

$$
f(z)=z+\sum_{n=2}^{\infty} a_{n} z^{n} \quad(z \in \mathbb{U}: \mathbb{U}=\{z \in \mathbb{C}:|z|<1\}) .
$$

For $h(z) \in \mathcal{A}$, given by $h(z)=z+\sum_{n=2}^{\infty} h_{n} z^{n}$, the Hadamard product (or convolution) of $f(z)$ and $h(z)$ is defined by:

$$
(f * h)(z)=z+\sum_{n=2}^{\infty} a_{n} h_{n} z^{n}=(h \times f)(z) .
$$

Definition 1 ([1, 2], and [3] with $\mathbf{p}=1)$. Let $\mathcal{P}_{k}^{\lambda}(\rho) \quad(0 \leq \rho<1, k \geq 2$ and $\left.|\lambda|<\frac{\pi}{2}\right)$ denote the class of functions $p(z)=1+\sum_{n=1}^{\infty} c_{n} z^{n}$, which are analytic in $\mathbb{U}$ and satisfy the conditions:

(i) $p(0)=1$

$$
\text { (ii) } \int_{0}^{2 \pi}\left|\frac{\Re\left\{e^{i \lambda} p(z)\right\}-\rho \cos \lambda}{1-\rho}\right| \leq k \pi \cos \lambda\left(r<1, z=r e^{i \theta} \in \mathbb{U}\right) .
$$

We note that:

(i) $\mathcal{P}_{k}^{\lambda}(0)=\mathcal{P}_{k}^{\lambda}\left(k \geq 2\right.$ and $\left.|\lambda|<\frac{\pi}{2}\right)$ is the class of functions introduced by Robertson (see [4]), and he derived a variational formula for functions in this class.

(c) The Author(s). 2019 Open Access This article is distributed under the terms of the Creative Commons Attribution 4.0 International License (http://creativecommons.org/licenses/by/4.0/), which permits unrestricted use, distribution, and reproduction in any medium, provided you give appropriate credit to the original author(s) and the source, provide a link to the Creative Commons license, and indicate if changes were made. 
(ii) $\mathcal{P}_{k}^{0}(\rho)=\mathcal{P}_{k}(\rho)(0 \leq \rho<1, k \geq 2)$ is the class of functions introduced by Padmanabhan and Parvatham [5] (see also Umarani and Aouf [6]).

(iii) $\mathcal{P}_{k}^{0}(0)=\mathcal{P}_{k}(k \geq 2)$ is the class of functions having their real parts bounded in the mean on $\mathbb{U}$, introduced by Robertson [4] and studied by Pinchuk [7].

(iv) $\mathcal{P}_{2}^{0}(\rho)=\mathcal{P}(\rho)(0 \leq \rho<1)$ is the class of functions with positive real part of order $\rho, 0 \leq \rho<1$.

(v) $\mathcal{P}_{2}^{0}(0)=\mathcal{P}$ is the class of functions having positive real part for $z \in \mathbb{U}$.

By the Koebe one-quarter theorem [8], we know that the image of $\mathbb{U}$ under every univalent function $f \in \mathcal{A}$ contains the disk with center in the origin and radius $1 / 4$. Therefore, every univalent function $f$ has an inverse $f^{-1}$ satisfies:

$$
f^{-1}(f(z))=z(z \in \mathbb{U}) \text { and } f\left(f^{-1}(w)\right)=w\left(|w|<r_{0}(f), r_{0}(f) \geq 1 / 4\right) .
$$

It is easy to see that the inverse function has the form:

$$
f^{-1}(w)=w-a_{2} w^{2}+\left(2 a_{2}^{2}-a_{3}\right) w^{3}-\left(5 a_{2}^{3}-5 a_{2} a_{3}+a_{4}\right) w^{4}+\ldots .
$$

A function $f \in \mathcal{A}$ is said to be bi-univalent in $\mathbb{U}$ if both $f$ and its inverse map $g=f^{-1}$ are univalent in $\mathbb{U}$.

Let $\sum$ denote the class of bi-univalent functions in $\mathbb{U}$ in the form (1). For interesting examples about the class $\sum$, see [9].

The object of this paper is to introduce new subclass of Bazilevič functions [10] for the class $\sum$ with bounded boundary rotation and defined by using convolution as follows:

Definition 2 Let $f, h \in \sum, \alpha \in \mathbb{C}^{*}, \beta \geq 0,0 \leq \rho<1, k \geq 2$ and $|\lambda|<\frac{\pi}{2}$, then $(f * h)(z) \in \sum$ is said to be in the class $\mathcal{M}_{\alpha, \lambda, \rho, k, \beta}(f * h)$ if it satisfies the following conditions:

$$
\left\{(1-\alpha)\left(\frac{(f * h)(z)}{z}\right)^{\beta}+\alpha \frac{z(f * h)^{\prime}(z)}{(f * h)(z)}\left(\frac{(f * h)(z)}{z}\right)^{\beta}\right\} \in \mathcal{P}_{k}^{\lambda}(\rho)(z \in \mathbb{U})
$$

and

$$
\left\{(1-\alpha)\left(\frac{(f * h)^{-1}(w)}{w}\right)^{\beta}+\alpha \frac{w\left((f * h)^{-1}(w)\right)^{\prime}}{(f * h)^{-1}(w)}\left(\frac{(f * h)^{-1}(w)}{w}\right)^{\beta}\right\} \in \mathcal{P}_{k}^{\lambda}(\rho)(w \in \mathbb{U}) .
$$

We note that by putting different values for $h, \alpha, \beta, k, \lambda$, and $\rho$, in the above definition, we have:

(1) $\mathcal{M}_{1,0, \rho, k, \beta}\left(f \times \frac{z}{1-z}\right)=R_{\sum}(\rho, k, \beta)\left(f \in \sum, \beta \geq 0,0 \leq \rho<1, k \geq 2\right)$ (see [11], with $\gamma=1$ );

(2) $\mathcal{M}_{\alpha, 0, \rho, k, 1}(f * h)=\mathcal{L}_{\alpha, \rho, k}(f * h)\left(f, h \in \sum, \alpha \in \mathbb{C}^{*}, 0 \leq \rho<1, k \geq 2\right)$ (see [12]);

(3) $\mathcal{M}_{\eta, 0, \rho, 2,1}(f * h)=\mathcal{L}_{\eta, \rho}(f * h)\left(f, h \in \sum, \eta \geq 0,0 \leq \rho<1\right)$ (see [13] and [14]);

(4) $\mathcal{M}_{\eta, 0, \rho, 2,1}\left(f \times \frac{z}{1-z}\right)=\mathcal{L}_{\eta, \rho}(f)(z)\left(f \in \sum, \eta \geq 0,0 \leq \rho<1\right)$ (see [15]);

(5) $\mathcal{M}_{1,0, \rho, 2, \beta}\left(f \times \frac{z}{1-z}\right)=\mathcal{L}_{\rho, \beta}(f)(z)\left(f \in \sum, \beta \geq 0,0 \leq \rho<1\right)$ (see [16]);

(6) $\mathcal{M}_{1,0, \rho, 2,1}\left(f \times \frac{z}{1-z}\right)=\mathcal{L}_{\rho}(f)(z)\left(f \in \sum, 0 \leq \rho<1\right)$ (see [9]); 
(7) $\mathcal{M}_{\alpha, 0, \rho, 2, \beta}\left(f \times \frac{z}{1-z}\right)=\mathcal{N} \mathcal{P}_{\sum}^{\beta, \alpha}(0, \rho)\left(f \in \sum, \beta, \alpha \geq 0,0 \leq \rho<1\right)$ (see [[17], with $\beta=0]$ );

(8) $\mathcal{M}_{1,0, \rho, 2, \beta}\left(f \times \frac{z}{1-z}\right)=\mathcal{R}_{\sum}(\beta, \rho)\left(f \in \sum, \beta \geq 0,0 \leq \rho<1\right)$ (see [18]).

Also, we can obtain the following subclasses:

(i) $\mathcal{M}_{\alpha, \lambda, \rho, k, \beta}\left(f \times \frac{z}{1-z}\right)=\digamma_{\alpha, \lambda, \rho, k, \beta}(f)$

$$
=\left\{f \in \sum:(1-\alpha)\left(\frac{f(z)}{z}\right)^{\beta}+\alpha \frac{z f^{\prime}(z)}{f(z)}\left(\frac{f(z)}{z}\right)^{\beta} \in \mathcal{P}_{k}^{\lambda}(\rho)\right.
$$

and $\left.(1-\alpha)\left(\frac{f^{-1}(w)}{w}\right)^{\beta}+\alpha \frac{w\left(\left(f^{-1}(w)\right)^{\prime}\right.}{f^{-1}(w)}\left(\frac{f^{-1}(w)}{w}\right)^{\beta} \in \mathcal{P}_{k}^{\lambda}(\rho)\right\}$;

(ii) $\mathcal{M}_{\alpha, 0, \rho, k, \beta}(f * h)=\mathcal{F}_{\alpha, \rho, k, \beta}(f * h)$

$$
\begin{aligned}
& \quad=\left\{f, h \in \sum:(1-\alpha)\left(\frac{(f * h)(z)}{z}\right)^{\beta}+\alpha \frac{z(f * h)^{\prime}(z)}{(f * h)(z)}\left(\frac{(f * h)(z)}{z}\right)^{\beta} \in \mathcal{P}_{k}(\rho)\right. \\
& \text { and } \left.(1-\alpha)\left(\frac{(f * h)^{-1}(w)}{w}\right)^{\beta}+\alpha \frac{w\left((f * h)^{-1}(w)\right)^{\prime}}{(f * h)^{-1}(w)}\left(\frac{(f * h)^{-1}(w)}{w}\right)^{\beta} \in \mathcal{P}_{k}(\rho)\right\} ;
\end{aligned}
$$

(iii) $\mathcal{M}_{\alpha, 0, \rho, 2, \beta}(f * h)=\mathcal{F}_{\alpha, \rho, \beta}(f * h)$

$$
\begin{aligned}
& \quad=\left\{f, h \in \sum: \Re\left[(1-\alpha)\left(\frac{(f * h)(z)}{z}\right)^{\beta}+\alpha \frac{z(f * h)^{\prime}(z)}{(f * h)(z)}\left(\frac{(f * h)(z)}{z}\right)^{\beta}\right]>\rho\right. \\
& \text { and } \left.\Re\left[(1-\alpha)\left(\frac{(f * h)^{-1}(w)}{w}\right)^{\beta}+\alpha \frac{w\left((f * h)^{-1}(w)\right)^{\prime}}{(f * h)^{-1}(w)}\left(\frac{(f * h)^{-1}(w)}{w}\right)^{\beta}\right]>\rho\right\} ;
\end{aligned}
$$

(iv) $\mathcal{M}_{\alpha, \lambda, 0, k, \beta}(f * h)=\mathcal{M}_{\alpha, \lambda, k, \beta}(f * h)$

$$
\begin{aligned}
& \quad=\left\{f, h \in \sum:(1-\alpha)\left(\frac{(f * h)(z)}{z}\right)^{\beta}+\alpha \frac{z(f * h)^{\prime}(z)}{(f * h)(z)}\left(\frac{(f * h)(z)}{z}\right)^{\beta} \in \mathcal{P}_{k}^{\lambda}\right. \\
& \text { and } \left.(1-\alpha)\left(\frac{(f * h)^{-1}(w)}{w}\right)^{\beta}+\alpha \frac{w\left((f * h)^{-1}(w)\right)^{\prime}}{(f * h)^{-1}(w)}\left(\frac{(f * h)^{-1}(w)}{w}\right)^{\beta} \in \mathcal{P}_{k}^{\lambda}\right\} ;
\end{aligned}
$$

(v) $\mathcal{M}_{\alpha, 0,0, k, \beta}(f * h)=\mathcal{M}_{\alpha, k, \beta}(f * h)$

$$
\begin{aligned}
& \quad=\left\{f, h \in \sum:(1-\alpha)\left(\frac{(f * h)(z)}{z}\right)^{\beta}+\alpha \frac{z(f * h)^{\prime}(z)}{(f * h)(z)}\left(\frac{(f * h)(z)}{z}\right)^{\beta} \in \mathcal{P}_{k}\right. \\
& \text { and } \left.(1-\alpha)\left(\frac{(f * h)^{-1}(w)}{w}\right)^{\beta}+\alpha \frac{w\left((f * h)^{-1}(w)\right)^{\prime}}{(f * h)^{-1}(w)}\left(\frac{(f * h)^{-1}(w)}{w}\right)^{\beta} \in \mathcal{P}_{k}\right\} ;
\end{aligned}
$$

(vi) $\mathcal{M}_{\alpha, 0,0,2, \beta}(f * h)=\mathcal{M}_{\alpha, \beta}(f * h)$

$$
\begin{aligned}
& \quad=\left\{f, h \in \sum:(1-\alpha)\left(\frac{(f * h)(z)}{z}\right)^{\beta}+\alpha \frac{z(f * h)^{\prime}(z)}{(f * h)(z)}\left(\frac{(f * h)(z)}{z}\right)^{\beta} \in \mathcal{P}\right. \\
& \text { and } \left.(1-\alpha)\left(\frac{(f * h)^{-1}(w)}{w}\right)^{\beta}+\alpha \frac{w\left((f * h)^{-1}(w)\right)^{\prime}}{(f * h)^{-1}(w)}\left(\frac{(f * h)^{-1}(w)}{w}\right)^{\beta} \in \mathcal{P}\right\} ;
\end{aligned}
$$


(vii) $\mathcal{M}_{1, \lambda, \rho, k, \beta}(f * h)=\mathbb{F}_{\lambda, \rho, k, \beta}(f * h)$

$$
\begin{aligned}
= & \left\{f, h \in \sum: \frac{z(f * h)^{\prime}(z)}{(f * h)(z)}\left(\frac{(f * h)(z)}{z}\right)^{\beta} \in \mathcal{P}_{k}^{\lambda}(\rho)\right. \text { and } \\
& \left.\frac{w\left((f * h)^{-1}(w)\right)^{\prime}}{(f * h)^{-1}(w)}\left(\frac{(f * h)^{-1}(w)}{w}\right)^{\beta} \in \mathcal{P}_{k}^{\lambda}(\rho)\right\} ;
\end{aligned}
$$

or

$$
=\left\{f \in \sum: \frac{e^{i \lambda\left[\frac{z(f * h)^{\prime}(z)}{(f * h)(z)}\left(\frac{(f * h)(z)}{z}\right)^{\beta}\right]-\rho \cos \lambda-i \sin \lambda}}{(1-\rho) \cos \lambda} \in \mathcal{P}_{k}\right.
$$

and $\left.\frac{e^{i \lambda}\left[\frac{z(f * h)^{\prime}(z)}{(f * h)(z)}\left(\frac{(f * h)(z)}{z}\right)^{\beta}\right]-\rho \cos \lambda-i \sin \lambda}{(1-\rho) \cos \lambda} \in \mathcal{P}_{k}\right\}$;

(viii) $\mathcal{M}_{1,0, \rho, 2, \beta}(f * h)=\mathbb{F}_{\rho, \beta}(f * h)$

$$
\begin{aligned}
& =\left\{f, h \in \sum: \Re\left[\frac{z(f * h)^{\prime}(z)}{(f * h)(z)}\left(\frac{(f * h)(z)}{z}\right)^{\beta}\right]>\rho\right. \\
& \text { and } \left.\Re\left[\frac{w\left((f * h)^{-1}(w)\right)^{\prime}}{(f * h)^{-1}(w)}\left(\frac{(f * h)^{-1}(w)}{w}\right)^{\beta}\right]>\rho\right\} .
\end{aligned}
$$

In order to obtain our main results, we have to recall here the following lemma.

Lemma 1 ([3] with $\mathbf{p}=1)$. If $p(z)=1+\sum_{n=1}^{\infty} c_{n} z^{n} \in \mathcal{P}_{k}^{\lambda}(\rho)$, then

$$
\left|c_{n}\right| \leq(1-\rho) k \cos \lambda .
$$

The result is sharp. Equality is attained for the odd coefficients and even coefficients respectively for the functions:

$$
\begin{aligned}
& p_{1}(z)=1+(1-\rho) \cos \lambda e^{-i \lambda}\left[\left(\frac{k+2}{4}\right)\left(\frac{1-z}{1+z}\right)-\left(\frac{k-2}{4}\right)\left(\frac{1+z}{1-z}\right)-1\right], \\
& p_{2}(z)=1+(1-\rho) \cos \lambda e^{-i \lambda}\left[\left(\frac{k+2}{4}\right)\left(\frac{1-z^{2}}{1+z^{2}}\right)-\left(\frac{k-2}{4}\right)\left(\frac{1+z^{2}}{1-z^{2}}\right)-1\right] .
\end{aligned}
$$

We note that for $\lambda=0$ in Lemma 1, we obtain the result obtained by Goswami et al. [19] [Lemma 2.1] for the class $\mathcal{P}_{k}(\rho)$.

In this paper, we will obtain the coefficients bounds $\left|a_{2}\right|$ and $\left|a_{3}\right|$ for the class $\mathcal{M}_{\alpha, \lambda, \rho, k, \beta}(f * h)$, which defined in Definition 2 .

\section{Coefficient estimates for functions in the class $\mathcal{M}_{\boldsymbol{\alpha}, \lambda, \rho, k, \beta}(\boldsymbol{f} * \boldsymbol{h})$}

Theorem 1 Let $f, h \in \sum, \alpha \in \mathbb{C}^{*} \backslash\left\{-1, \frac{-1}{2}\right\}, \beta \geq 0,0 \leq \rho<1, k \geq 2,|\lambda|<\frac{\pi}{2}$, $f * h$ given by (2) and $h_{2}, h_{3} \neq 0$. Iff $* h$ belongs to $\mathcal{M}_{\alpha, \lambda, \rho, k, \beta}(f * h)$, then:

$$
\left|a_{2}\right| \leq \min \left\{\sqrt{\frac{2 k(1-\rho) \cos \lambda}{|2 \alpha+\beta|(\beta+1)\left|h_{2}\right|^{2}}} ; \frac{k(1-\rho) \cos \lambda}{|\alpha+\beta|\left|h_{2}\right|}\right\}
$$

and 


$$
\left|a_{3}\right| \leq \frac{k(1-\rho) \cos \lambda}{|2 \alpha+\beta|\left|h_{3}\right|}+\frac{[k(1-\rho) \cos \lambda]^{2}}{|\alpha+\beta|^{2}\left|h_{3}\right|} .
$$

The result is sharp.

Proof 1 If $(f * h) \in \mathcal{M}_{\alpha, \lambda, \rho, k, \beta}(f * h)$, then from Definition 2, we have:

$$
(1-\alpha)\left(\frac{(f * h)(z)}{z}\right)^{\beta}+\alpha \frac{z(f * h)^{\prime}(z)}{(f * h)(z)}\left(\frac{(f * h)(z)}{z}\right)^{\beta}=p(z), p \in \mathcal{P}_{k}^{\lambda}(\rho)
$$

and

$$
(1-\alpha)\left(\frac{(f * h)^{-1}(w)}{w}\right)^{\beta}+\alpha \frac{w\left((f * h)^{-1}(w)\right)^{\prime}}{(f * h)^{-1}(w)}\left(\frac{(f * h)^{-1}(w)}{w}\right)^{\beta}=q(w), q \in \mathcal{P}_{k}^{\lambda}(\rho),
$$

where $p$ and $q$ have Taylor expansions as follows:

$$
\begin{aligned}
& p(z)=1+p_{1} z+p_{2} z^{2}+p_{3} z^{3}+\ldots, z \in \mathbb{U}, \\
& q(w)=1+q_{1} w+q_{2} w^{2}+q_{3} w^{3}+\ldots ., w \in \mathbb{U} .
\end{aligned}
$$

By comparing the coefficients in (11) with (13) and coefficients in (12) with (14), we obtain:

$$
\begin{aligned}
& p_{1}=(\beta+\alpha) a_{2} h_{2}, \\
& p_{2}=(\beta+2 \alpha) a_{3} h_{3}+\frac{(\beta+2 \alpha)(\beta-1)}{2} a_{2}^{2} h_{2}^{2}, \\
& q_{1}=-(\beta+\alpha) a_{2} h_{2}
\end{aligned}
$$

and

$$
q_{2}=(\beta+2 \alpha)\left(2 a_{2}^{2} h_{2}^{2}-a_{3} h_{3}\right)+\frac{(\beta+2 \alpha)(\beta-1)}{2} a_{2}^{2} h_{2}^{2} .
$$

Since $p, q \in \mathcal{P}_{k}^{\lambda}(\rho)$ and by applying Lemma 1, we have:

$$
\left|p_{n}\right| \leq k(1-\rho) \cos \lambda(n \geq 1)
$$

and

$$
\left|q_{n}\right| \leq k(1-\rho) \cos \lambda(n \geq 1) .
$$

From (16) and (18) and using inequalities (19) and (20), we obtain:

$$
\left|a_{2}\right|^{2} \leq \frac{1}{|2 \alpha+\beta||\beta+1|} \frac{\left|p_{2}\right|+\left|q_{2}\right|}{\left|h_{2}\right|^{2}} \leq \frac{2 k(1-\rho) \cos \lambda}{|2 \alpha+\beta|(\beta+1)\left|h_{2}\right|^{2}} .
$$

Also, from (15) and (19), we obtain:

$$
\left|a_{2}\right| \leq \frac{k(1-\rho) \cos \lambda}{|\alpha+\beta|\left|h_{2}\right|} .
$$

Subtracting (18) from (16), we have:

$$
p_{2}-q_{2}=2(2 \alpha+\beta)\left(a_{3} h_{3}-a_{2}^{2} h_{2}^{2}\right) .
$$

Also, we have:

$$
p_{1}^{2}+q_{1}^{2}=2(\alpha+\beta)^{2} a_{2}^{2} h_{2}^{2} .
$$


After using (23), (24), (19), and (20), and some easily calculations, we obtain:

$$
\left|a_{3}\right| \leq \frac{k(1-\rho) \cos \lambda}{|2 \alpha+\beta|\left|h_{3}\right|}+\frac{[k(1-\rho) \cos \lambda]^{2}}{|\alpha+\beta|^{2}\left|h_{3}\right|},
$$

which completes the proof of Theorem 1. The result is sharp in view of the fact that assertion (8) of Lemma 1 is sharp.

Remark 1 For $h(z)=\frac{z}{1-z}, \beta=\alpha=1, k=2$, and $\lambda=0$ in Theorem 1, we obtain the result obtained by Srivastava et al. [9] [Theorem 2].

Putting $h(z)=\frac{z}{1-z}$ in Theorem 1, we obtain the following corollary.

Corollary 1 Let $f \in \sum, \alpha \in \mathbb{C}^{*} \backslash\left\{-1, \frac{-1}{2}\right\}, \beta \geq 0,0 \leq \rho<1, k \geq 2$ and $|\lambda|<\frac{\pi}{2}$. If $f \in \digamma_{\alpha, \lambda, \rho, k, \beta}(f)$, then:

$$
\left|a_{2}\right| \leq \min \left\{\sqrt{\frac{2 k(1-\rho) \cos \lambda}{|2 \alpha+\beta|(\beta+1)}} ; \frac{k(1-\rho) \cos \lambda}{|\alpha+\beta|}\right\}
$$

and

$$
\left|a_{3}\right| \leq \frac{k(1-\rho) \cos \lambda}{|2 \alpha+\beta|}+\frac{[k(1-\rho) \cos \lambda]^{2}}{|\alpha+\beta|^{2}} .
$$

The result is sharp.

Putting $\lambda=0$ in Theorem 1, we obtain the following corollary.

Corollary 2 Let $f, h \in \sum, \alpha \in \mathbb{C}^{*} \backslash\left\{-1, \frac{-1}{2}\right\}, \beta \geq 0,0 \leq \rho<1, k \geq 2, f * h$ given by (2) and $h_{2}, h_{3} \neq 0$. Iff $* h \in \mathcal{F}_{\alpha, \rho, k, \beta}(f * h)$, then:

$$
\left|a_{2}\right| \leq \min \left\{\sqrt{\frac{2 k(1-\rho)}{|2 \alpha+\beta|(\beta+1)\left|h_{2}\right|^{2}}} ; \frac{k(1-\rho)}{|\alpha+\beta|\left|h_{2}\right|}\right\}
$$

and

$$
\left|a_{3}\right| \leq \frac{k(1-\rho)}{|2 \alpha+\beta|\left|h_{3}\right|}+\frac{[k(1-\rho)]^{2}}{|\alpha+\beta|^{2}\left|h_{3}\right|} .
$$

The result is sharp.

Putting $\lambda=0$ and $k=2$ in Theorem 1, we obtain the following corollary.

Corollary 3 Let $f, h \in \sum, \alpha \in \mathbb{C}^{*} \backslash\left\{-1, \frac{-1}{2}\right\}, \beta \geq 0,0 \leq \rho<1, f * h$ given by (2) and $h_{2}, h_{3} \neq 0$. Iff $* h \in \mathcal{F}_{\alpha, \rho, \beta}(f * h)$, then:

$$
\left|a_{2}\right| \leq \min \left\{\sqrt{\frac{4(1-\rho)}{|2 \alpha+\beta|(\beta+1)\left|h_{2}\right|^{2}}} ; \frac{2(1-\rho)}{|\alpha+\beta|\left|h_{2}\right|}\right\}
$$

and

$$
\left|a_{3}\right| \leq \frac{2(1-\rho)}{|2 \alpha+\beta|\left|h_{3}\right|}+\frac{[2(1-\rho)]^{2}}{|\alpha+\beta|^{2}\left|h_{3}\right|} .
$$

The result is sharp.

Putting $\alpha=1$ in Theorem 1 , we obtain the following corollary. 
Corollary 4 Let $f, h \in \sum, \beta \geq 0,0 \leq \rho<1, k \geq 2,|\lambda|<\frac{\pi}{2}, f * h$ given by (2) and $h_{2}, h_{3} \neq 0$. Iff $* h \in \mathbb{F}_{\lambda, \rho, k, \beta}(f * h)$, then:

$$
\left|a_{2}\right| \leq \min \left\{\sqrt{\frac{2 k(1-\rho) \cos \lambda}{(2+\beta)(\beta+1)\left|h_{2}\right|^{2}}} ; \frac{k(1-\rho) \cos \lambda}{(1+\beta) h_{2}}\right\}
$$

and

$$
\left|a_{3}\right| \leq \frac{k(1-\rho) \cos \lambda}{(2+\beta)\left|h_{3}\right|}+\frac{[k(1-\rho) \cos \lambda]^{2}}{(1+\beta)^{2}\left|h_{3}\right|} .
$$

The result is sharp.

Putting $\alpha=1, k=2$, and $\lambda=0$ in Theorem 1, we obtain the following corollary.

Corollary 5 Let $f, h \in \sum, \beta \geq 0,0 \leq \rho<1, f * h$ given by (2) and $h_{2}, h_{3} \neq 0$. If $f * h \in \mathbb{F}_{\rho, \beta}(f * h)$, then:

$$
\left|a_{2}\right| \leq \min \left\{\sqrt{\frac{4(1-\rho)}{(2+\beta)(\beta+1)\left|h_{2}\right|^{2}}} ; \frac{2(1-\rho)}{(1+\beta) h_{2}}\right\}
$$

and

$$
\left|a_{3}\right| \leq \frac{2(1-\rho)}{(2+\beta)\left|h_{3}\right|}+\frac{[2(1-\rho)]^{2}}{(1+\beta)^{2}\left|h_{3}\right|} .
$$

The result is sharp.

Putting $\rho=0$ in Theorem 1 , we obtain the following corollary.

Corollary 6 Let $f, h \in \sum, \alpha \in \mathbb{C}^{*} \backslash\left\{-1, \frac{-1}{2}\right\}, \beta \geq 0,|\lambda|<\frac{\pi}{2}, k \geq 2, f * h$ given by (2) and $h_{2}, h_{3} \neq 0$. Iff $* h \in \mathcal{M}_{\alpha, \lambda, k, \beta}(f * h)$, then:

$$
\left|a_{2}\right| \leq \min \left\{\sqrt{\frac{2 k \cos \lambda}{|2 \alpha+\beta|(\beta+1)\left|h_{2}\right|^{2}}} ; \frac{k \cos \lambda}{|\alpha+\beta|\left|h_{2}\right|}\right\}
$$

and

$$
\left|a_{3}\right| \leq \frac{k \cos \lambda}{|2 \alpha+\beta|\left|h_{3}\right|}+\frac{[k \cos \lambda]^{2}}{|\alpha+\beta|^{2}\left|h_{3}\right|} .
$$

The result is sharp.

Putting $\rho=\lambda=0$ in Theorem 1, we obtain the following corollary.

Corollary 7 Let $f, h \in \sum, \alpha \in \mathbb{C}^{*} \backslash\left\{-1, \frac{-1}{2}\right\}, \beta \geq 0, k \geq 2, f * h$ given by (2) and $h_{2}, h_{3} \neq 0$. Iff $* h \in \mathcal{M}_{\alpha, k, \beta}(f * h)$, then:

$$
\left|a_{2}\right| \leq \min \left\{\sqrt{\frac{2 k}{|2 \alpha+\beta|(\beta+1)\left|h_{2}\right|^{2}}} ; \frac{k}{|\alpha+\beta|\left|h_{2}\right|}\right\}
$$

and

$$
\left|a_{3}\right| \leq \frac{k}{|2 \alpha+\beta|\left|h_{3}\right|}+\frac{k^{2}}{|\alpha+\beta|^{2}\left|h_{3}\right|} .
$$

The result is sharp.

Putting $\rho=\lambda=0$ and $k=2$ in Theorem 1, we obtain the following corollary. 
Corollary 8 Let $f, h \in \sum, \alpha \in \mathbb{C}^{*} \backslash\left\{-1, \frac{-1}{2}\right\}, \beta \geq 0, f *$ hiven by (2) and $h_{2}, h_{3} \neq 0$. If $f * h \in \mathcal{M}_{\alpha, \beta}(f * h)$, then:

$$
\left|a_{2}\right| \leq \min \left\{\sqrt{\frac{4}{|2 \alpha+\beta|(\beta+1)\left|h_{2}\right|^{2}}} ; \frac{2}{|\alpha+\beta|\left|h_{2}\right|}\right\}
$$

and

$$
\left|a_{3}\right| \leq \frac{2}{|2 \alpha+\beta|\left|h_{3}\right|}+\frac{4}{|\alpha+\beta|^{2}\left|h_{3}\right|} .
$$

The result is sharp.

Putting $\lambda=0, \alpha=1$ and $h(z)=\frac{z}{1-z}$ in Theorem 1, we obtain the following corollary.

Corollary 9 Let $f \in \sum, 0 \leq \rho<1$ and $\beta \geq 0$. Iff $\in R_{\sum}(\rho, k, \beta)$, then:

$$
\left|a_{2}\right| \leq \min \left\{\sqrt{\frac{2 k(1-\rho)}{(2+\beta)(\beta+1)}} ; \frac{k(1-\rho)}{(1+\beta)}\right\}
$$

and

$$
\left|a_{3}\right| \leq \frac{k(1-\rho)}{(2+\beta)}+\frac{[k(1-\rho)]^{2}}{(1+\beta)^{2}} .
$$

The result is sharp.

Remark 2 The results in Corollary 9 correct the results obtained by Orhan et al. [11] [Theorem 2.11, with $\gamma=1$.].

Acknowledgements

The authors are grateful to the referees for their valuable suggestions.

Funding

Higher Institute for Engineering and Technology, New Damietta, Egypt

Availability of data and materials

Not applicable.

Authors' contributions

All authors jointly worked on the results, and they read and approved the final manuscript.

Competing interests

The authors declare that they have no competing interests.

\section{Publisher's Note}

Springer Nature remains neutral with regard to jurisdictional claims in published maps and institutional affiliations.

\section{Author details}

${ }^{1}$ Faculty of Science, Department of Mathematics, Mansoura University, Mansoura 35516, Egypt. ${ }^{2}$ Basic Sciences Department, Higher Institute for Engineering and Technology, New Damietta, Egypt.

Received: 19 April 2019 Accepted: 24 April 2019

Published online: 17 May 2019

\section{References}

1. Moulis, E. J.: Generalizations of the Robertson functions, Pacific. J. Math. 81(1), 167-174 (1979)

2. Noor, K., Arif, M., Muhammad, A.: Mapping properties of some classes of analytic functions under an integral operator. J. Math. Inequal. 4(4), 593-600 (2010)

3. Aouf, M. K.: A generalization of functions with real part bounded in the mean on the unit disc. Math. Japonica. 33(2), 175-182 (1988)

4. Robertson, M. S.: Variational formulas for several classes of analytic functions. Math. Z. 118, 311-319 (1976)

5. Padmanabh, K. S., Paravatham, R.: Properties of a class of functions with bounded boundary rotation. Ann. Polon. Math. 31(3), 842-853 (1975) 
6. Umarani, P. G., Aouf, M. K.: Linear combination of functions of bounded boundary rotation of order $\alpha$, Tamkang. J. Math. 20(1), 83-86 (1989)

7. Pinchuk, B.: Functions of bounded boundary rotation, Isr. J. Math. 10, 7-16 (1971)

8. Duren, P. L.: Univalent Functions. In: Grundlehren der Mathematischen Wissenschaften Series, p. 259. Springer Verlag, New York, (1983)

9. Srivastava, H. M., Mishra, A. K., Gochhayat, P.: Certain subclasses of analytic and bi-univalent functions, Appl. Math. Lett. 23, 1188-1192 (2010)

10. Bazilevič, I. E.: On a case of integrability in quadratures of the Lowner-Kufarev equation, Mat. Sb. 37(79), 471-476 (1955)

11. Orhan, H., Magesh, N., Balaji, V. K.: Certain classes of bi-univalent functions with bounded boundary variation, Tbilisi. Math. J. 10(4), 17-27 (2017)

12. Altınkaya, S., Yalçın, S.: Coefficient problem for certain subclasses of bi-univalent functions defined by convolution. Math. Moravica. 20(2), 15-21 (2016)

13. El-Ashwah, R. M.: Subclasses of bi-univalent functions defined by convolution. J. Egypt. Math. Soc. 22, 348-351 (2013)

14. Motamednezhad, A.: S. Nosratil and S. Zaker, Bounds for initial Maclaurin coefficients of a subclass of bi-univalent functions associated with subordination, Commun. Fac. S.i. Univ. Ank. Ser. A1 Math. Stat. 68(1), 125-135 (2019)

15. Frasin, B. A., Aouf, M. K.: New subclasses of bi-univalent functions, Appl. Math. Lett. 24(9), 1569-1573 (2011)

16. Prema, S., Keerthi, B. S.: Coefficient bounds for certain subclasses of analytic functions. J. Math. Anal. 4(1), 22-27 (2013)

17. Orhan, H., Magesh, N., Balaji, V. K.: Initial coefficient bounds for a general class of bi-univalent functions. Filomat. 29(6), 1259-1267 (2015)

18. Magesh, N., T.Rosy, Varma, S.: Coefficient estimate problem for a new subclass of bi-univalent functions. J. Compl. Anal. 2013, 3 (2013). Article ID 474231

19. Goswami, P., Alkahtani, B. S., Bulboaca, T.: Estimate for initial Maclaurin coefficients of certain subclasses of bi-univalent functions (2015). arXiv:1503.04644v1 [math.CV]

\section{Submit your manuscript to a SpringerOpen ${ }^{\circ}$ journal and benefit from:}

- Convenient online submission

- Rigorous peer review

- Open access: articles freely available online

- High visibility within the field

- Retaining the copyright to your article

Submit your next manuscript at $\boldsymbol{\triangleright}$ springeropen.com 